\title{
Clinical and molecular aspects of distal renal tubular acidosis in children
}

Martine TP Besouw ${ }^{1}$, Marc Bienias ${ }^{2}$, Patrick Walsh ${ }^{3}$, Robert Kleta ${ }^{1,4}$, William G van't Hoff', Emma Ashton ${ }^{4}$, Lucy Jenkins, ${ }^{4}$ Detlef Bockenhauer ${ }^{1,5}$

${ }^{1}$ Department of Pediatric Nephrology, Great Ormond Street Hospital for Children NHS Foundation Trust, London, UK

2 Department of Pediatrics, Medical Faculty Carl Gustav Carus, Technical University Dresden, Germany

${ }^{3}$ Great North Children's Hospital, Newcastle upon Tyne NHS Foundation Trust, Newcastle, UK

${ }^{4}$ North East Thames Regional Genetics Service Laboratories, Great Ormond Street Hospital for Children NHS Foundation Trust, London, UK

${ }^{5}$ Centre for Nephrology, University College London Institute of Child Health, London, UK

Corresponding author:

Prof. Dr. D. Bockenhauer

Department of Pediatric Nephrology

Great Ormond Street Hospital NHS Trust

Great Ormond Street

London WC1N 3JH

Tel: +442074059200

detlef.bockenhauer@gosh.nhs.uk 


\section{Abstract}

Background: Distal renal tubular acidosis (dRTA) is characterized by hyperchloremic metabolic acidosis, hypokalemia, hypercalciuria and nephrocalcinosis. It is due to reduced urinary acidification by the $\alpha$-intercalated cells in the collecting duct and can be caused by mutations in ATP6V1B1, ATP6V0A4, or SLC4A1. Treatment with alkali is the mainstay of therapy.

Patients and methods: This study presents clinical data on long-term follow-up of children with $\mathrm{dRTA}$ in a single centre, including genetic analysis.

Results: Twenty-four children were included. Genetic diagnosis was confirmed in 19 patients: 6 had mutations in ATP6V1B1, 10 in ATP6VOA4 and 3 in SLC4A1, 5 had no molecular diagnosis. There were 5 novel mutations (2 in ATP6V1B1 and 3 in ATP6VOA4). Two thirds of patients presented with features of proximal tubular dysfunction, leading to an erroneous diagnosis of renal Fanconi syndrome. The proximal tubulopathy disappeared after resolution of acidosis, indicating the importance of following proximal tubular function to establish the correct diagnosis. Growth retardation with a height below -2 SDS was found in 10 patients at presentation, but remained present in only 3 once established on alkali treatment. Sensorineural hearing loss was found in 5/6 patients with ATP6V1B1 mutations. Only 1 patient with ATP6VOA4 mutations had sensorineural hearing loss during childhood. Nine children developed medullary cysts, but without apparent clinical consequences. Cyst development in this cohort was not correlated with age at therapy onset, molecular diagnosis, growth parameters or renal function.

Conclusion: In general, the prognosis of dRTA is good in children treated with alkali.

\section{Key words}

Distal renal tubular acidosis, metabolic acidosis, hypokalemia, nephrocalcinosis, medullary cysts, gene mutation 


\section{Background}

Distal renal tubular acidosis (dRTA), also often referred to as type $1 \mathrm{RTA}$, is caused by a decreased capacity of the a-intercalated cells in the collecting duct to excrete protons. Biochemically, it is characterized by a hyperchloremic metabolic acidosis with normal plasma anion gap, hypokalemia and hypercalciuria with hypocitraturia causing nephrocalcinosis. Despite a profound metabolic acidosis, patients are unable to acidify their urine and will have a urinary $\mathrm{pH}>5.5[1,2]$.

Patients often present with vomiting, diarrhea or constipation, failure to thrive and/or rickets. Severe hypokalemia can cause muscle weakness or paralysis [2]. Chronic acidosis leads to bone demineralization which can result in rickets and/or stunted growth in children with dRTA. The excess of calcium that is released into the blood stream, combined with the decreased expression of renal calcium transporters in metabolic acidosis, causes hypercalciuria [3,4]. This hypercalciuria in turn can result in nephrocalcinosis, and if left untreated to renal calculi formation. Polyuria, due to impaired urinary concentrating ability (secondary nephrogenic diabetes insipidus) is another typical feature and may be related to electrolyte abnormalities in blood and/or urine and the nephrocalcinosis [5].

To date, several genes are known to cause dRTA. Autosomal recessive inheritance is noted in mutations in ATP6V1B1, ATP6VOA4, and sometimes SLC4A1. Mutations in the latter gene, however, generally cause the autosomal dominant form of dRTA [1].

In this study we present data on children with dRTA followed in our outpatient clinic over the last 20 years, including the available data of DNA analysis.

\section{Patients and methods}

This is a single centre, retrospective study on the molecular diagnosis and clinical follow-up of children with dRTA, followed in the specialized tubulopathy outpatient clinic at Great Ormond Street Hospital (London, UK) in the last 20 years. Distal renal tubular acidosis was defined as hyperchloremic metabolic acidosis with a normal plasma anion gap, with inappropriately high urinary $\mathrm{pH}$ on repeated sampling. All parents of children with $\mathrm{dRTA}$ were 
offered genetic analysis to confirm their diagnosis. Clinical and biochemical data were retrieved from the patients' medical records and included details until transition into adult services. Estimated glomerular filtration rate (eGFR) was measured using the bedside Schwartz formula [6].

\section{Results}

In total 24 patients were included, with a median follow-up of 10 years (range 1-18 years). Clinical details are presented in table 1. The molecular diagnosis was confirmed in 19/24 patients. Six patients had homozygous or compound heterozygous mutations in ATP6V1B1, 10 had mutations in ATP6VOA4 (including one pair of siblings) and 3 patients were found to have mutations in SLC4A1. We found 5 mutations not previously reported. These include 2 mutations in ATP6V1B1 (c.905G>C; p.(Arg302Pro) in patient 2 and c.490_499del; p.(Ile164Alafs*8) in patient 6) and 3 mutations in ATP6VOA4 (c.1312_1315del; p.(Asp438Metfs*13) in patient 9, c.970del; p.(Glu324Argfs $\left.{ }^{\star 22}\right)$ in patient 11 and c.1238G>A; p.(Gly413Asp) in patient 15). In patient 20 only one mutation was found in ATP6VOA4. Although this mutation was predicted to be pathogenic, he was not considered as having a confirmed molecular diagnosis since mutations in this gene have an autosomal recessive pattern of inheritance. Patients 5 and 15 were seen in our clinic for a second opinion, treatment strategies may therefore not necessarily represent the general practice in our hospital.

\section{Disease presentation}

Age at diagnosis

In general, patients with autosomal recessive mutations (patients 1-16 and patient 19) had a tendency to present earlier in life compared to patients with an autosomal dominant form of dRTA (patients 17 and 18). The children with autosomal recessive inherited mutations presented at a median age of 3 months (range 2 weeks -2 years and 2 months), only 3 out of 17 children presented after the age of 1 year. In contrast, both children with autosomal 
dominant mutations presented at after the age of 1 year, at 1 year and 5 months and 4 years, respectively (figure 1). This difference was statistically significant $(p<0.01$, student T-test).

Interestingly, patient 17 was assessed initially at the age of 1 year because of the family history of dRTA in both mother and maternal grandmother. Molecular diagnosis was not available in the family at the time and the initial clinical assessment was unremarkable with normal plasma electrolytes $\left(\mathrm{K}_{+}=4.3 \mathrm{mmol}, \mathrm{HCO} 3=20 \mathrm{mmol} / \mathrm{l}\right)$, normal venous $\mathrm{pH}$ (7.34), normocalciuria (calcium/creatinine ratio of $0.36 \mathrm{mmol} / \mathrm{mmol}$ ) and normal renal ultrasound. A diagnosis was not established at the time and only further observation recommended. At 17-months of age, she had an episode of diarrhea and subsequent blood tests revealed acidosis (venous $\mathrm{pH} 7.29$, plasma $\mathrm{HCO} 316 \mathrm{mmol} / \mathrm{l}$ ), with inappropriately elevated urine $\mathrm{pH}$ (7.5), revealing her to be affected, later confirmed by molecular diagnosis.

\section{Presenting symptoms}

Many children with dRTA presented with failure to thrive and growth retardation. This was most profound in the children with ATP6V1B1 mutations and in those in whom no genetic mutation could be found (figure 2). Severe growth retardation according to the first documented measurement after presentation, with a height on or below -2 standard deviations scores (SDS), was found in a total of 12 children. It was documented in 4/6 children with mutations in ATP6V1B1, in 3/10 children with mutations in ATP6V0A4, in 1/3 with SLC4A1 and in 4/5 children without a confirmed genetic diagnosis.

All children were found to have metabolic acidosis at initial presentation, but given the retrospective nature of this study the exact blood $\mathrm{pH}$ at diagnosis could only be recovered in 9 patients; all were $<7.25$. Serum bicarbonate levels at diagnosis, however, could be recovered in the majority of patients and were well below $18 \mathrm{mmol} / \mathrm{L}$. Both blood $\mathrm{pH}$ and serum bicarbonate levels were the lowest in children with mutations in ATP6V0A4, children with mutations in SLC4A1 generally presented with less severe acidosis (figure 3).

Two thirds of our patients presented with features of partial renal Fanconi syndrome. Fourteen children had tubular proteinuria, 8 children had a decreased tubular resorption of 
phosphate (TRP) and 5 patients showed mild generalized aminoaciduria. None of the children had glycosuria. Surprisingly, at first presentation, only 7/24 children had an elevated urinary calcium/creatinine ratio. However, these measurements were performed on spot urine samples, and patients were polyuric, which in our laboratory can result in inaccuracies in the determination of creatinine in urine, as the assay is optimized for higher creatinine concentrations. A formal 24-h urine calcium determination would have been more informative, but is difficult to obtain in non-toilet trained children without catherization.

All patients except for patient 17 had nephrocalcinosis at first presentation, the first ultrasound in patient 5 was performed abroad and the report could not be retrieved. Patient 17 had a family history of autosomal dominant dRTA (in her mother and grandmother) and was screened before the onset of symptoms. In the other children, at the moment of diagnosis nephrocalcinosis was reported by the radiologist to be mild (increased echogenicity in the borders of the pyramids) in 10 patients, moderate (diffuse increased echogenicity of the entire pyramids) or moderate to marked in 4 children and marked (homogenous increased echogenicity of the entire pyramids, often in the presence of acoustic shadowing) in another 8 patients. Only 3 children developed renal calculi (patient 10, 19 and 23), none of them required medical intervention. There was no correlation with the degree of nephrocalcinosis at first presentation (marked in patient 10 and 23 and mild in patient 19).

\section{$\underline{\text { Treatment }}$}

Treatment with alkali (bicarbonate and/or citrate) was started at a median age of 4 months (range 2 weeks - 5 years). Initial alkali prescription was as high as $8.6 \mathrm{mmol}$ bicarbonate equivalent $/ \mathrm{kg} /$ day in some children at the onset of therapy in order to achieve normal plasma bicarbonate values. In general, most children had a higher alkali requirement in the first years of life, which decreased after the first decade. Moreover, children with mutations that encoded part of the vacuolar $\mathrm{H}^{+}$-ATPase (ATP6V1B1 and ATP6V0A4) were prescribed 
higher alkali doses compared to children with mutations that encode the anion exchanger 1 (SLC4A1), (figure 4).

Thiazide diuretics were administered to 3 patients. In patient 8 thiazides were started at the age of 2 months and stopped after two years, in patient 9 thiazides were started at the age of 1 month and stopped one month later. In both of them, thiazides were started in the referring centres. Patient 23 was started at the age of 11 years after two episodes of flank pain that resolved after passing concrements, Thiazide treatment was continued into adulthood.

Patient 15 was started on indomethacin by the referring team at the age of 6 months because of severe polyuria with the possible diagnosis of renal Fanconi syndrome. After the diagnosis of dRTA the indomethacin had been stopped at the age of 2 years, but was restarted because of worsening of polyuria.

\section{$\underline{\text { Clinical features at last follow-up }}$}

\section{Growth parameters}

After the start of alkali treatment, there was a clear improvement in growth parameters in most children. Sustained growth retardation, with a height on or below -2 SDS on the most recent measurement, was documented in only 3 children, while the overall height SDS had improved in all groups. A similar improvement in weight SDS was found (figure 2). There was no clear correlation with growth retardation at the most recent follow-up and the age of therapy onset (2 months, 9 months and 5.3 years, respectively) or gene mutation (ATP6V0A4, SLC4A1 and unknown). One child with sustained growth retardation had a normal renal function with an eGFR of $152 \mathrm{~mL} / \mathrm{min} / 1.73 \mathrm{~m}^{2}$, the other two had chronic kidney disease (CKD) stage 2 with an eGFR of 67 and $70 \mathrm{~mL} / \mathrm{min} / 1.73 \mathrm{~m}^{2}$, respectively.

\section{Chronic kidney disease}

Renal insufficiency was found in 9/24 children, all of them had an eGFR of $60-90$ $\mathrm{mL} / \mathrm{min} / 1.73 \mathrm{~m}^{2}$, consistent CKD stage 2. There was no clear correlation with renal function 
and genetic diagnosis: decreased renal function was found in 1/6 patients with mutations in ATP6V1B1, in 5/10 patients with ATP6V0A4 mutations, in $1 / 3$ patients with SLC4A1 mutations and in 2/5 children who had no confirmed genetic diagnosis.

\section{Radiological features}

In 9/24 patients, medullary cysts were reported on the most recent renal ultrasound. The age at which the first cysts were noted ranged between 3-17 years. Two out of three patients who developed renal calculi had medullary cysts, but there was no correlation with the degree of nephrocalcinosis at diagnosis (which was mild in 5 patients with medullary cysts, moderate in 1 and marked in another 3 patients). Also, there was no clear effect on renal function, which

was normal in 3 patients with renal cysts while the remaining 6 patients had CKD stage 2. Four out of ten patients with ATP6VOA4 mutations developed cysts, all after the age of 10 . Two out of three patients with mutations in SLC4A1 developed cysts, one was 4 years old and the other at the age of 17 . No molecular diagnosis could be made in the remaining 3 patients that developed medullary cysts, all of them were below the age of 10 when the cysts were first noted. There was no apparent correlation between the degree of nephrocalcinosis and the development of CKD.

\section{Hearing}

Hearing loss was documented in 11 children, including all children with ATP6V1B1 mutations. The hearing problems were found to be sensorineural in origin in 6 patients, 5 of which had documented mutations in ATP6V1B1. In the other child no causative mutations were found. Three children were diagnosed with conductive hearing loss. This was attributed to recurrent ear infections in 2 of them and improved over time, in the other child no mutation was found. Another 2 children were found to have combined sensorineural and conductive hearing problems, one of them had a mutation in ATP6VOA4 and the other one in SLC4A1.

\section{Discussion}


We present clinical data on the follow-up of children with dRTA in a single centre, including the available data on genetic analysis. Although the sample size is rather small, this study contains valuable data on the long-term follow-up of patients with a rare disease. The majority of patients were followed for a long period of time, 20/24 patients were followed for at least 5 years and 12/24 patients for at least 10 years. The addition of molecular diagnostics enables us to compare the clinical phenotype with the molecular genotype. Moreover, the analysis of clinical data informs the prognosis and management of patients with this rare disease.

Lastly, we found 5 mutations that to our knowledge are new to the literature. Publication of these will help establishing the diagnosis in other patients found to carry these mutations.

\section{$\underline{\text { Treatment }}$}

The administration of alkali is the cornerstone of treatment of dRTA. Due to a higher metabolic rate and protein intake in growing children, they may need relatively higher treatment doses than adults when corrected for body weight to maintain a normal $\mathrm{pH}$. This was previously reported in a historical study in which 5 children with dRTA were followed for a period of $<10$ years [7]. Where adults in general require $0.5-1 \mathrm{mEq} / \mathrm{kg} / \mathrm{d}$ of bicarbonate, this can be up to 5-8 mEq/kg in children [1]. The fact that younger children in general need higher doses of alkali was confirmed in this study. Moreover, children with mutations interrupting the function of the vacuolar $\mathrm{H}^{+}$-ATPase generally needed higher doses of alkali compared to those with mutations in SLC4A1 (figure 4) and prescribed doses were typically highest at therapy onset. This is in line with the finding that children with mutations in ATP6VOA4 in general had a lower blood $\mathrm{pH}$ and lower serum bicarbonate levels at diagnosis (figure 3 ). In cases with severe hypokalemia, potassium supplements may also be warranted. In these patients, the prescription of potassium citrate or potassium bicarbonate can prove to be an elegant way to administer both. 
Thiazide diuretics are sometimes used to decrease urinary calcium excretion, but they can in turn increase the risk of hypokalemia and polyuria and are thus not routinely used in our centre. In our cohort of 24 patients, only 3 children developed small calculi and none of them needed an intervention. Moreover, urinary calcium excretion is increased in acidosis and decreases after the administration of alkali $[7,8]$. This makes it a good marker for adherence to and/or adequacy of treatment, but is no longer reliable after the administration of thiazide diuretics. Thus, in our opinion the use of alkali treatment, sometimes with additional potassium supplements, is sufficient to treat the majority of children with dRTA.

The experience with patient 17 , in whom routine biochemistries of plasma and urine were normal at the age of 12 months, suggests that a clinical diagnosis of dRTA cannot be excluded at such a young age in at-risk children and should ideally be established molecularly, or, if this not possible, by clinical examination when the system is "stressed" (for instance after acid loading or, as in patient 17, during an episode of gastroenteritis with intestinal bicarbonate loss).

\section{Associated symptoms}

Interestingly, 16/24 children in this study had features of renal Fanconi syndrome at presentation. The latter indicates a proximal tubular dysfunction characterized by lowmolecular weight proteinuria (also referred to as tubular proteinuria), generalized aminoaciduria, hyperphosphaturia, glucosuria and increased urinary losses of bicarbonate (causing metabolic acidosis). Several other solutes that are reabsorbed in the proximal tubule can be lost as well $[9,10]$. In our cohort, the 16 patients with partial renal Fanconi syndrome showed a combination of low-molecular weight proteinuria (measured as urinary retinol binding protein/creatinine ratio), increased urinary losses of phosphate (measured as TRP) and/or mild generalized aminoaciduria. Metabolic acidosis was obviously found in all patients, since this is a key feature of dRTA. However, none of the patients had a full-blown renal Fanconi syndrome, since none of them showed glucosuria. In this way, the proximal tubulopathy resembles that of Lowe syndrome or Dent disease [11]. After initiation of alkali 
treatment, the features of renal Fanconi syndrome disappeared in all patients, establishing the correct diagnosis of dRTA in some children that had previously been diagnosed with renal Fanconi syndrome of unknown etiology. There have been several reports of children with dRTA presenting with partial renal Fanconi syndrome (low-molecular weight proteinuria, hyperphosphaturia and/or generalized aminoaciduria). As in our study, none of them had glucosuria and in all of them the proximal tubulopathy disappeared after treatment onset [7,12-14]. The exact mechanism by which dRTA causes proximal tubular damage is unknown, but given the similarity of the tubulopathy with Dent Disease/Lowe syndrome it is tempting to speculate that this may be due to impairment of similar pathways. Indeed, apart from those with unknown mutations the proximal tubulopathy was seen only in children with mutations in subunits of the vacuolar ATPase, which is expressed together with CLC-5 (the chloride transporter underlying Dent disease) in the lysosomes of the proximal tubule and $\alpha$ intercalated cells [15]. However, why this lysosomal dysfunction would improve with correction of the acidosis is unclear, and the proximal tubulopathy may just reflect a general dysfunction from the acidosis. From a clinical point of view, the key conclusion is to not prematurely exclude dRTA as a potential diagnosis in patients with a proximal tubulopathy, especially in young children with associated nephrocalcinosis. After correction of the acidosis and hypokalemia the abnormal markers of proximal tubular function should be monitored, since they will normalize in children with underlying dRTA.

In our population of 24 children with dRTA, the majority of patients (16 in total) had proven mutations in ATP6V1B1 $(\mathrm{n}=6)$ and ATP6VOA4 $(\mathrm{n}=10)$, interrupting the function of the vacuolar $\mathrm{H}^{+}$-ATPase. Both mutations can be associated with sensorineural hearing loss [1]. In fact, all 6 patients in our cohort who were found to have ATP6V1B1 mutations also had hearing loss. However, this was conductive in origin in one of them and was attributed to recurrent ear infections. Only one child with mutations in ATP6VOA4 was diagnosed with hearing loss: patient 13 was found to have a combined bilateral sensorineural and unilateral conductive hearing loss. Her sister, however, was not noted to have any hearing problems. Since both have the same mutation in ATP6VOA4, it remains unclear whether this mutation is 
in fact causing the hearing loss in patient 13. It has been noted before that hearing loss in children with ATP6V1B1 mutations generally presents before the age of 10 years, while hearing loss in patients with ATP6VOA4 mutations is often only diagnosed from the second decade of life $[16,17]$. The outcome in our cohort is in line with these findings. It also warrants the need for regular hearing examination in patients without any signs of deafness at diagnosis, since sensorineural hearing loss can still occur later in life.

We present one child (patient 19) with the concomitant occurrence of Southeast Asian ovalocytosis and dRTA due to mutations in SLC4A1. The protein encoded by this gene is a polypeptide which forms a homodimer, to form the transporter AE1. The first deletion found in this patient is c.1199_1225del; p.(Ala400-Ala408del). This is a well-known mutation typically causing Southeast Asian ovalocytosis, in general occurring without a renal phenotype. Mutant non-functioning polypeptides form heterodimers with the wild type polypeptide. The minimal net effect on the transport function of the newly formed protein results in the absence of a renal phenotype in presence of a wild type allele. However, this patient had a second mutation in her other allele, being c.2102G $>A$; p.(Gly701Asp). This mutation gives rise to a mutant polypeptide that is trapped within the cell (probably in the Golgi apparatus), thus causing autosomal recessive dRTA. The presence of both mutations in 1 patient (one resulting in a non-functioning polypeptide and the other one resulting in a polypeptide being trapped intracellularly) causes autosomal recessive dRTA, while the c.1199_1225del mutation alone has been reported not to cause a renal phenotype $[18,19]$. Although very rare, this combination of c.1199_1225del with c.2102G>A has been reported before to cause a combination of Southeast Asian ovalocytosis and dRTA [20].

In this study, we found medullary cysts on the most recent renal ultrasound in $37.5 \%$ of our study population (9/24 patients). The development of medullary cysts in a subset of patients with dRTA has been well described [21]. The exact etiology of these cysts remains unclear, but again it was hypothesized that this may be caused by hypokalemic nephropathy. Besides the ischemic changes, hypokalemic nephropathy is associated with tubular hypertrophy and dilatation, which may cause renal cyst formation [22]. However, not all 
conditions with chronic hypokalemia result in cyst formation. For instance, Bartter syndrome is often associated with severe chronic hypokalemia but medullary cysts are only rarely reported [23]. Moreover, in our population all patients had developed cysts while they were receiving treatment. There was no correlation with the development of medullary cysts and the degree of nephrocalcinosis at diagnosis, nor with renal function at last follow-up. The development of medullary cysts is thus noted in a subset of patients with dRTA, but the clinical consequences remain limited in our cohort.

\section{$\underline{\text { Conclusion }}$}

In general, the prognosis of dRTA is good when the diagnosis can be made early and if there is a good compliance to alkali treatment [2]. In this study, we did not find a correlation between the age at therapy onset or gene mutation and stunted growth or renal function. Nine patients were found to have CKD stage 2. Deterioration of renal function can be seen in patients with dRTA, mainly in those with hypercalciuria and recurrent renal stones, but progression into end-stage renal disease is only rarely reported [24]. Growth retardation is often noted at diagnosis in children with chronic dRTA and is attributed to chronic metabolic acidosis. However, after the initiation of alkaline treatment catch-up growth can be noted $[2,7]$ and in this study most children (87.5\%) had a height within normal limits for age at last follow-up, despite profound failure to thrive at diagnosis in $50 \%$ of them (figure 2 ). Thus, we can confirm the general good prognosis of dRTA in children under alkali treatment.

In conclusion, we present clinical data on long-term follow-up of 24 children with dRTA in a single center, including available data on genetic screening and on 5 novel disease causing mutation. All but 1 patient presented with the combination of metabolic acidosis and nephrocalcinosis, 16 children had features of proximal tubular dysfunction which disappeared after initiation of treatment. The diagnosis of renal Fanconi syndrome should be made with care in children with severe metabolic acidosis and nephrocalcinosis at a young age; markers of proximal tubulopathy should be followed after initiation of treatment. Failure to thrive is often noted at diagnosis, alkali treatment results in improved growth 
parameters in most children. Although their clinical relevance remains uncertain, medullary cysts were found in 6 children and are often reported in patients with dRTA. Also, hearing loss is often associated with $\mathrm{dRTA}$, mainly when mutations are documented in ATP6V1B1 or ATP6VOA4. In the latter gene, sensorineural deafness often develops in the second decade of life, warranting long-term follow-up of hearing in children with dRTA with normal initial hearing screening who have mutations in ATP6V1B1, ATP6VOA4 or in whom no mutation was found. The basic treatment of dRTA consists in the prescription of alkali, if necessary combined with potassium supplementation. Although there was a tendency in children with ATP6V1B1 or ATP6VOA4 mutations to present earlier in life and to need higher doses of alkali, our study population was rather small and we found no statistical significant correlation between gene mutation, age at therapy onset, growth parameters or renal function. Longterm studies including genetic analysis in a bigger population, including data before and after transition into adult care can further elaborate on the effects of early treatment onset and gene defect on final height, deterioration of renal function over time and the development of nephrocalcinosis with calculi and medullary cysts.

\section{Acknowledgements}

Funding for this study was kindly provided by the European Union, FP7 (grant agreement 2012-305608 "European Consortium for High-Throughput Research in Rare Kidney Diseases (EURenOmics) and by the British Kidney Patient Association (BKPA). 


\section{References}

1. Batlle D, Haque SK (2012) Genetic causes and mechanisms of distal renal tubular acidosis. Nephrol Dial Transplant 27:3691-3704

2. Escobar L, Mejia N, Gil H, Santos F (2013) Distal renal tubular acidosis: a hereditary disease with an inadequate urinary $\mathrm{H}(+)$ excretion. Nefrologia 33:289-296

3. Lemann J, Jr., Gray RW, Maierhofer WJ, Cheung HS (1986) The importance of renal net acid excretion as a determinant of fasting urinary calcium excretion. Kidney Int 29:743746

4. Nijenhuis T, Renkema KY, Hoenderop JG, Bindels RJ (2006) Acid-base status determines the renal expression of $\mathrm{Ca} 2+$ and $\mathrm{Mg} 2+$ transport proteins. $\mathrm{J} \mathrm{Am} \mathrm{Soc}$ Nephrol 17:617-626

5. Bockenhauer D, Bichet DG (2013) Inherited secondary nephrogenic diabetes insipidus: concentrating on humans. Am J Physiol Renal Physiol 304:F1037-1042

6. Schwartz GJ, Muñoz A, Schneider MF, Mak RH, Kaskel F, Warady BA, Furth SL (2009) New equations to estimate GFR in children with CKD. J Am Soc Nephrol 20:629-637

7. Rodriguez-Soriano J, Vallo A, Castillo G, Oliveros R (1982) Natural history of primary distal renal tubular acidosis treated since infancy. J Pediatr 101:669-676

8. Ariceta G, Vallo A, Rodriguez-Soriano J (2004) Acidosis increases magnesiuria in children with distal renal tubular acidosis. Pediatr Nephrol 19:1367-1370

9. Bökenkamp A, Ludwig M (2011) Disorders of the renal proximal tubule. Nephron Physiol 118:p1-6

10. Klootwijk ED, Reichold M, Unwin RJ, Kleta R, Warth R, Bockenhauer D (2015) Renal Fanconi syndrome: taking a proximal look at the nephron. Nephrol Dial Transplant $30: 1456-1460$

11. Bockenhauer D, Bökenkamp A, van't Hoff W, Levtchenko E, Kist-van Holthe JE, Tasic V, Ludwig M (2008) Renal phenotype in Lowe Syndrome: a selective proximal tubular dysfunction. Clin J Am Soc Nephrol 3:1430-1436 
12. Tasic V, Korneti P, Gucev Z, Hoppe B, Blau N, Cheong HI (2008) Atypical presentation of distal renal tubular acidosis in two siblings. Pediatr Nephrol 23:1177-1181

13. Igarashi T, Kawato H, Kamoshita S (1990) Reversible low-molecular-weight proteinuria in patients with distal renal tubular acidosis. Pediatr Nephrol 4:593-596

14. Watanabe $T$ (2005) Proximal renal tubular dysfunction in primary distal renal tubular acidosis. Pediatr Nephrol 20:86-88

15. Günther W, Lüchow A, Cluzeaud F, Vandewalle A, Jentsch TJ (1998) CIC-5, the chloride channel mutated in Dent's disease, colocalizes with the proton pump in endocytotically active kidney cells. Proc Natl Acad Sci U S A 95:8075-8080

16. Smith AN, Skaug J, Choate KA, Nayir A, Bakkaloglu A, Ozen S, Hulton SA, Sanjad SA, Al-Sabban EA, Lifton RP, Scherer SW, Karet FE (2000) Mutations in ATP6N1B, encoding a new kidney vacuolar proton pump 116-kD subunit, cause recessive distal renal tubular acidosis with preserved hearing. Nat Genet 26:71-75

17. Stover EH, Borthwick KJ, Bavalia C, Eady N, Fritz DM, Rungroj N, Giersch AB, Morton CC, Axon PR, Akil I, Al-Sabban EA, Baguley DM, Bianca S, Bakkaloglu A, Bircan Z, Chauveau D, Clermont MJ, Guala A, Hulton SA, Kroes H, Li Volti G, Mir S, Mocan H, Nayir A, Ozen S, Rodriguez Soriano J, Sanjad SA, Tasic V, Taylor CM, Topaloglu R, Smith AN, Karet FE (2002) Novel ATP6V1B1 and ATP6V0A4 mutations in autosomal recessive distal renal tubular acidosis with new evidence for hearing loss. J Med Genet 39:796-803

18. Alper SL (2009) Molecular physiology and genetics of Na+-independent SLC4 anion exchangers. J Exp Biol 212:1672-1683

19. Wrong O, Bruce LJ, Unwin RJ, Toye AM, Tanner MJ (2002) Band 3 mutations, distal renal tubular acidosis, and Southeast Asian ovalocytosis. Kidney Int 62:10-19

20. Bruce LJ, Wrong O, Toye AM, Young MT, Ogle G, Ismail Z, Sinha AK, McMaster P, Hwaihwanje I, Nash GB, Hart S, Lavu E, Palmer R, Othman A, Unwin RJ, Tanner MJ (2000) Band 3 mutations, renal tubular acidosis and South-East Asian ovalocytosis in 
Malaysia and Papua New Guinea: loss of up to $95 \%$ band 3 transport in red cells. Biochem J 350 Pt 1:41-51

21. Igarashi T, Shibuya K, Kamoshita S, Higashihara E, Kawato H, Hagishima K, Kosugi T (1991) Renal cyst formation as a complication of primary distal renal tubular acidosis. Nephron 59:75-79

22. Toback FG, Ordónez NG, Bortz SL, Spargo BH (1976) Zonal changes in renal structure and phospholipid metabolism in potassium-deficient rats. Lab Invest 34:115-124

23. Watanabe T, Tajima T (2005) Renal cysts and nephrocalcinosis in a patient with Bartter syndrome type III. Pediatr Nephrol 20:676-678

24. Jha V, Muthukumar T, Singh SK, Kohli HS, Sud K, Gupta KL, Sakhuja V (1999) Familial distal renal tubular acidosis. Nephrol Dial Transplant 14:1334-1335 
Figure 1. Age at onset of alkali treatment.

Boxplot showing the age in months when alkali treatment was first initiated, in the different genotypes on the left and of all patients on the right. 
Figure 2. Height and weight at diagnosis and at most recent follow-up.

Boxplot showing the height SDS (upper panels) and weight SDS (lower panels) at diagnosis (left panels) and at most recent follow-up (right panels). In each panel the different genotypes are depicted on the left and the mean of all patients is depicted on the right. 
Figure 3. Blood pH and serum bicarbonate levels at diagnosis.

Boxplot showing blood $\mathrm{pH}$ (left panel) and serum bicarbonate (right panel) at diagnosis. In each panel the different genotypes are depicted on the left and the mean of all patients is depicted on the right. 
Figure 4. Alkali treatment (equivalent to mmols of bicarbonate/kg/day) at different ages.

Boxplot showing the alkali requirement at the age of $1,5,10$ and 15 years. The first four panels represent the alkali requirement per genotype, the last panel represents the alkali requirement in all patients. 